\title{
Human-animal interspecies chimerism via blastocyst complementation: advances, challenges and perspectives: a narrative review
}

\author{
Yuhang Li ${ }^{1,2,3}$, Ke Huang ${ }^{1,3}$ \\ ${ }^{1}$ The Seventh Affiliated Hospital, Sun Yat-sen University, Shenzhen, China; ${ }^{2}$ University of Chinese Academy of Sciences, Beijing, China; ${ }^{3}$ CAS Key \\ Laboratory of Regenerative Biology, Guangzhou Institutes of Biomedicine and Health, Chinese Academy of Sciences, Guangzhou, China \\ Contributions: (I) Conception and design: Both authors; (II) Administrative support: None; (III) Provision of study materials or patients: None; \\ (IV) Collection and assembly of data: Both authors; (V) Data analysis and interpretation: Both authors; (VI) Manuscript writing: Both authors; (VII) \\ Final approval of manuscript: Both authors. \\ Correspondence to: Ke Huang. The Seventh Affiliated Hospital, Sun Yat-sen University, 628 Zhenyuan Road, Guangming Street, Guangming New \\ District, Shenzhen 518107, China. Email: huangk65@mail.sysu.edu.cn.
}

Objective: Interspecific human-animal chimerism via blastocyst complementation provides a promising
strategy to generate function human cells, tissues or organs from human pluripotent stem cells (hPSCs),
although it is still quite challenging. In this review, we will mainly focus on the recent advances, such as the
options of donor hPSCs and the understanding of interspecific chimera barriers, challenges, and perspectives
on the efficient generation of human-animal interspecies chimeras. Background: hPSCs, including the human embryonic stem cells (hESCs) and the human induced pluripotent stem cells (hiPSCs) hold great promise for regenerative medicine to treat various degenerative diseases. However, although hPSCs can differentiate to all lineage cells in dish, the functionality of these cells is limited, hinting that the in vitro differentiation system failed to fully recapture the in vivo development. A promising alternative strategy is in vivo generation of functional human cells in animals through interspecies chimerism, based on the principle that mammalian development is highly conserved across species. This strategy was inspired by the successful generation of functional rat pancreas in mice through blastocyst injection of rat pluripotent stem cells (PSCs). Over the past ten years, since this milestone work was reported, advances have been made in the human-animal interspecies chimerism. However, it is still challenging to efficiently generate human cells, tissues, or organs in the interspecies chimeras. This phenomenon suggests that there are still obstacles to illustrate and overcome implicated in human-animal interspecies chimeras.

Methods: Narrative overview of the literatures reported the recent advances, challenges and perspectives regarding the interspecies chimerism via blastocyst complementation.

Conclusions: Human-animal interspecies chimerism via blastocyst complementation is a valuable method to generate functional human cells, tissues or organs, while there are at least three barriers need to be overcome. Firstly, conventional hPSCs should be converted to possess the chimera competency; secondly, efficient human-animal chimerism are required to robustly generate human derivatives in chimera; thirdly, the discrepancy regarding the developmental regulation network between human and host animals must be eliminated to generate certain human cells, tissues or organs in the interspecies chimeras.

Keywords: Human pluripotent stem cell (hPSC); regenerative medicine; interspecies chimeras; barrier

Received: 21 December 2020; Accepted: 28 June 2021; Published: 11 October 2021.

doi: $10.21037 /$ sci-2020-074

View this article at: https://dx.doi.org/10.21037/sci-2020-074 


\section{Introduction}

Human pluripotent stem cells (hPSCs), including the human embryonic stem cells (hESCs) and the human induced pluripotent stem cells (hiPSCs), are characterized by their capacities of unlimited proliferation and differentiation into all lineage cells. Therefore, the hPSCs hold great potential for the treatment of various degenerative diseases. The hESCs were initially derived from the preimplantation blastocysts over twenty years ago (1). In contrast, it is until recently reported that the hESCs could also be generated through somatic cell nuclear transfer (SCNT) from adult cells with low efficiency (2). The clinical application of hESCs is implicated with several concerns, such as the destruction of human embryos to generate hESCs and allogeneic immune rejection of the hESCs derived transplants. In contrast, the hiPSCs reprogramed from the patient's somatic cells could bypass the challenges of the hESCs and open the door for personalized regenerative medicine. With the availability of the hESCs and hiPSCs, efforts have been dedicated to developing in vitro strategies to differentiate the hPSCs into mature and functional cells, tissues, and even organs for regenerative treatments.

However, the advancement in obtaining functional cells from hPSCs has been limited. To date, only retinal cells (3) and nature killer cells (4) derived from hPSCs showed function similarity to their in vivo counterparts. The maturation and function of other cell types from the hPSCs, like hematopoietic stem/progenitor cells $(5,6)$, liver cells (7) and pancreas beta cells (8-10) were compromised. The failure of in vitro protocols in generating mature and functional cells from hPSCs suggesting that the in vitro differentiation failed to fully recapture the in vivo development. Thereby, a novel strategy to overcome this issue is desirable.

Notably, pluripotent stem cells (PSCs) possess a privilege called chimera formation. They could contribute to host animal development after injection into preimplantation embryos, making it possible to generate functional cells from PSCs by chimera formation. This concept was proved by a milestone work reported in 2010, which described the functional rat pancreas generation in the $P d x 1$ gene knockout mouse through blastocyst injection of rat PSCs (11). This report highlighted a potential alternative to generate mature and functional human cells in interspecies chimeras through blastocyst complementation (Figure 1). Yet, to achieve this goal, at least three major challenges have to be overcome: firstly, conventional hPSCs must be converted to possess the chimera competency; secondly, high efficiency of the human-animal chimerism are required to robustly generate human cells in chimera; thirdly, the discrepancy regarding the developmental regulation network between human and host animals must be eliminated to generate certain types of mature and functional human derivatives in the interspecies chimeras. In this review, we will focus on the recent advances and challenges on the human-animal interspecies chimerism via blastocyst complementation. Other approaches to acquire interspecies chimeras at the blastocyst stage, like aggregation of cleavage stage embryos, will not be discussed here due to ethical concerns. We present the following article in accordance with the narrative review reporting checklist (available at https:// dx.doi.org/10.21037/sci-2020-074).

\section{Blastocyst complementation}

Blastocyst complementation was a method utilizing the PSCs' chimera capacity to generate donor-derived cells, tissues, or organs by injecting the PSC into recipient morulas/blastocysts with corresponding cell, tissue, or organ development deficiency. This method was first introduced using wild type (WT) mouse embryonic stem cells (mESCs) to complement Rag2-deficient recipient mouse blastocysts. Since the Rag2-deficient mice lacked the $\mathrm{T}$ and $\mathrm{B}$ lymphocyte, these cells in the chimeras should be exclusively derived from the WT mESCs (12). This assay has been used to evaluate the lymphocyte potential of the Gata2-deficient mESCs (13). Later, this method was used to complement the Pdx1 (14) and Fab (15)-deficient mouse blastocysts with WT mESCs and mouse induced pluripotent stem cells (miPSCs) respectively. The WT mESCs/ miPSCs contributed to the pancreas and liver development in chimeras. A landmark report in 2010 by Hiromitsu Nakauchi's group proved the feasibility of generating xenoorgan through blastocyst complementation. They used rat PSCs as a donor to complement the $P d x 1$-deficient mouse blastocysts. As a result, the rat PSCs populated the entire pancreas epithelium in the $P d x 1$-deficient adult mice, and the pancreas functionally maintained the serum glucose levels in the chimera (11). Consistently, in a reverse experiment, the mESCs also contribute to functional islet in the $P d x 1$-null rat host (16). In addition, the rat PSCs also showed the capability to form thymus in nude mice through blastocyst complementation (17). The success of rat-mouse interspecies chimerism raised the possibility of generating functional human cells in host animals. However, to fulfill 


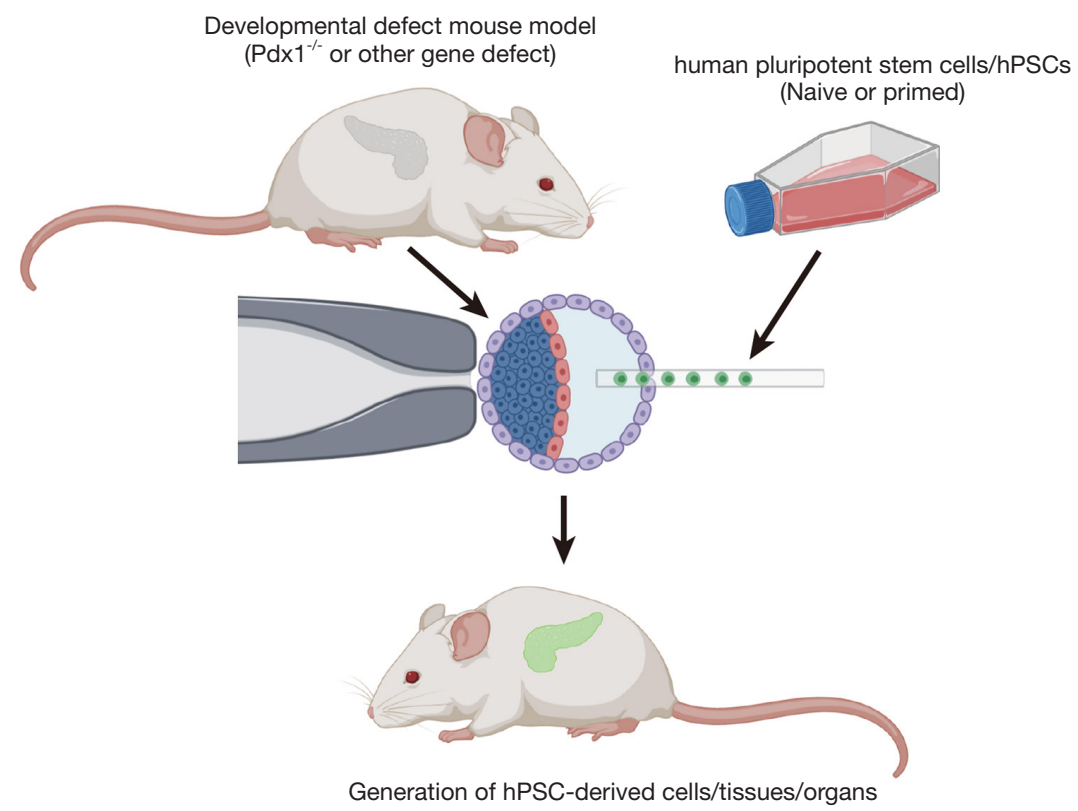

Figure 1 Schematic representation of interspecies chimeras from human pluripotent stem cells via blastocyst complementation. In brief, the hPSCs were injected into the host embryos to rescue their cell, tissue or organ developmental defects, such $P d x 1^{-/}$mouse with pancreas development failure. The survived $P d x 1^{-/-}$mice were supposed to harbor the hPSCs derived pancreases. hPSC, human pluripotent stem cell.

this goal, the conventional hPSCs must be converted to be with chimera competency in blastocysts.

\section{Cellular status of PSCs}

Conventional hPSCs were considered to be in a primed state. They resemble the mouse epiblast stem cells (EpiSCs) regarding cultural requirements and transcriptional profile, while the mESCs were in a naïve state (18). The primed and naïve state represents two different pluripotent states and harbors distinct development potential in chimera formation. Indeed, the naïve mESCs can integrate to the preimplantation blastocysts and contribute to the three germ layers during subsequent development. On the contrary, the primed hPSCs and EpiSCs failed to integrate into the blastocysts. However, they can integrate into the postimplantation embryos(19,20). Due to chimera formation in postimplantation embryos always involved the sacrifice of host embryos. Hence, it is presumed that the conventional primed hPSCs should be converted into naïve or naive-like states to endow them with chimera potential in the blastocysts.

Many groups have reported attempts to convert the primed to naïve or naïve like states of hPSCs. For instance, Gafni et al. reported that primed hPSCs could be transformed into a "naïve" state with chimera potential in mice (21). In addition, Takashima et al. (22) and Theunissen et al. $(23,24)$ established a ground/naive state of hPSCs, respectively, which also showed contribution of human cells in mouse morulas/blastocysts or embryos. Plus, Wu et al. introduced a distinct naïve like state, enabling the chimera potential of hPSCs in pig but not mouse (25). Besides, Yang et al converted the primed human PSCs into extended PSCs (EPSCs), which also possessed the potency to contribute to embryonic and extraembryonic tissues in mice (26). Recently, $\mathrm{Hu}$ et al reported a naïve state of hPSCs with robust chimera formation as quantification by the next-generation sequencing (NGS) of $18 S \mathrm{rDNA}$ in the chimera (27). However, human cell contribution in the interspecies chimeras is still relatively low in these reported human-animal chimeras. These observations highlighted that the naïve state of hPSCs is not sufficient for efficient chimera formation in the interspecific blastocysts. Hence, barriers in interspecies chimerism of hPSCs must be fully illustrated.

\section{Apoptosis is the initial barrier in interspecies chimerism of hPSCs}

In 2016, Masaki et al. reported that mouse EpiSCs injected into blastocysts rapidly disappeared because of apoptosis, and induced BCL2 expression enabled the mouse EpiSCs to 
survive in blastocysts and form chimeras (28). Remarkably, they showed that apoptosis inhibition could also enable primed rat EpiSCs to form interspecies chimeras in mouse blastocysts, overriding barriers regarding developmental stage and species (28). Wang et al. further proved that upon inhibition of apoptosis by BCL2 overexpression, hESCs could also contribute to mouse embryos' development (29). In addition, we reported that overexpression of BMI1 in hPSCs could also overcome the apoptosis and endowed the hPSCs with interspecies chimerism in both mouse, rabbit, and pig. More importantly, we demonstrated that the anti-apoptosis ability is also a prerequisite for naïve hPSCs to form chimera. The naïve hPSCs with high antiapoptosis competence possessed a high level of chimera formation efficiency (30). Recently, cell competition has been confirmed to be responsible to the apoptosis of primed hPSCs in the chimera, and overcoming the competition improved the survival and chimerism of human cells in early mouse embryos (31). These observations suggested that anti-apoptosis is a general property for cells that could form interspecies chimeras.

In particular, Das et al. injected hiPSCs with BCL2 overexpression into the ETV2-null pig blastocysts with endothelial deficiency and found that all endothelial cells were of human origin in the chimera (32), demonstrating that anti-apoptosis could enable the human tissue generation in the chimera. Notably, the author found that the number of chimeric embryos decreases over time after injection of BCL2 overexpressed hiPSCs. In comparison, the viable human cells in the embryos displayed a similar proliferation rate to the porcine blastomere cells delivered into the porcine parthenogenotes (32). These results suggested that there might be other hurdles, such as growth competition $(33,34)$, responsible for the initial reduction of human cell viability and decrease of human cell positive chimeras, which eventually led to the inefficient interspecies chimera formation of hPSCs.

\section{Xenobarrier implicated with the developmental niche discrepancy}

Except for the hurdles discussed above, the developmental niche discrepancy between human and animals should also be noted. The rationale of interspecies chimerism is based on the principle that the mammalian development is a highly conserved process across species. Thereby, the host niche, including stromal cells or signals, could support the donor cells' development. However, the successful reports on interspecific chimera have been limited in generating rat pancreas and thymus in mouse and human endothelial cells in pig embryos. Other cells, tissues, or organs like $\mathrm{T}$ cells, hematopoietic stem cells (HSCs), or liver have yet not been successfully generated in the interspecies chimeras even the rat-mouse chimeras. As Isotani et al. reported, rat ESCs could contribute to the thymus in nude mice through blastocyst complementation, while rat $\mathrm{T}$ cells could not be detected in the chimera. However, after transplanting the rat thymus from the chimera into the nude rat, $T$ cells can be generated in the recipient (17). These data suggested that the developmental niche in the mouse embryos is not sufficient for the rat T cell or HSC generation (17). Also, it is worth noting that the mESCs efficiently contributed to endothelial and hematopoietic cells in the mouse embryos with Flk-1 deficiency, and the chimera could survive to adulthood; otherwise, the Flk-1null embryo died at E8.5-E9.5. While, the Flk-1 deficient mouse embryos with rat ESCs contribution survived at E9.5, but unable to survive to E13.5, which might be due to immature vascularization of the rat derived endothelial cells or incomplete contribution of donor cells to the AGM region (35). Considering the human cells could proliferate well after initial integration into the host environment (32), the discrepancy of the developmental niche between different species might be a major xenobarrier for the successful generation of certain cell types in human-animal interspecies chimerism from hPSCs.

\section{Perspective: humanized hosts for interspecies chimerism}

There are unneglectable differences in the biological systems between human and animals. For instance, functional Toll-like receptor 10 (TLR10) is absent in mice (36). Also, human leukemia inhibitory factor (LIF) can activate mouse LIF receptor and be used for mESC maintenance. In contrast, mouse LIF cannot bind to human receptor, rendering mouse LIF inactive to human cells (37). In addition, the interaction of IL2 and IL2R across pig and human was defective, as porcine IL2 did not effectively induce human lymphocyte proliferation and human IL2 also had limited induction effect on porcine lymphocyte proliferation (38).

Thereby, for efficient generation of interspecies chimerism with hPSCs in animal embryos, it would be desirable to construct the humanized hosts for the chimera formation. The humanized hosts, like humanized mice, 
refer to the mice engrafted with human cells, tissues, or transgenic mice expressing human genes. Indeed, the humanized mice have been widely used to support human cells in vivo. For example, to enhance long-term human HSC engraftment, overexpression of membrane-bound human stem cell factor (SCF) $(39,40)$, and knockin of human thrombopoietin (TPO) (41) have been applied in mice. These approaches have increased the contribution of human blood cells in the host mice. Furthermore, through knockin of human M-CSF, IL3, GM-CSF, and TPO into their respective loci of immunodeficient $\mathrm{Rag}^{-{ }^{-/}} \mathrm{Il} 2 \mathrm{rg}^{-/-}$ mouse, the humanized mice (MISTRG) develop functional human innate immune cells, including monocytes/ macrophages and natural killer cell from engrafted human HSCs (42). Recently, by injection human liver cell into the $\mathrm{Fab}^{-1-}$ MISTRG mice, Song et al. proved that the liver humanized mice could enable the generation of circulating human red blood cells from engrafted human HSCs (43).

These reports highlighted not only the discrepancy between human and animal development regulation program, but also the significance of humanized animal in supporting human cell differentiation/development in host animals. Thus, it would be necessary to generate interspecies chimeras in humanized animals. To this end, firstly genes crucial for human cell development, but inactive or absent in the hosts must be fully illustrated. Then, the human genes should be transferred into their host loci through gene knockin or overexpression to support the human cell, tissue, or organ development. We believe that it will benefit human cell development and maturation in the interspecies chimeras through constructing the humanized hosts.

\section{Conclusions}

In this review, we summarized the advances and several hurdles in interspecies chimerism. As discussed, apoptosis is the first obstacle of hPSCs in interspecies chimerism, which could be a result of cell competition (31). The antiapoptosis ability is crucial for the chimera potential of both primed and naïve hPSCs (30). Besides, other hurdles, such as growth competition might be responsible for the inefficient interspecies chimerism of hPSCs even with anti-apoptosis ability $(33,34)$. In addition, another major hurdle of the human-animal interspecies chimerism is the developmental niche discrepancy between human and host animals; thereby humanized animals could be an ideal model to harvest specific human cells, tissues, or organs in the chimera such as HSCs, liver.

Also, the interspecies chimerism is still facing several other challenges as reviewed elsewhere (44-46), such as (I) high level of xenogenic cells in the chimera led to higher risk of abortion and malformation (16), though it is currently not observed in human animal chimeras; (II) lymphoid infiltration in the donor derived tissues in the chimera similar to the autoimmune diseases (16), calling for the use of immunodeficient host animals; (III) contribution of human cells to the germ cells or neural system, which might be overcome by deletion of critical genes involved in the germ cell or neural system development in the hPSCs (45); and (IV) host virus infection to the donor derived tissues, yet the virus genes can also be inactivated by gene editing (47).

Nevertheless, interspecies chimerism provided a promising method to generate functional cells, tissues, or even organs with three-dimensional structures from hPSCs. Thus, it could be possible to overcome donor shortages and treat patients on the waiting list for transplantation with degenerative diseases. Moreover, the interspecies chimerism could also be valuable to other research, such as stem cell fate determination, developmental biology, and disease modeling and drug screening. Therefore, the interspecies chimerism via blastocyst complementation should be significantly enhanced and would be beneficial to human society.

\section{Acknowledgments}

Funding: This work was supported by the Key Laboratory of Regenerative Biology, Guangzhou Institutes of Biomedicine and Health, Chinese Academy of Sciences (KLRB202001); the National Natural Science Foundation of China (32071454, 31801225).

\section{Footnote}

Reporting Checklist: The authors have completed the narrative review reporting checklist. Available at https:// dx.doi.org/10.21037/sci-2020-074

Conflicts of Interest: Both authors have completed the ICMJE uniform disclosure form (available at https://dx.doi. org/10.21037/sci-2020-074). The authors have no conflicts of interest to declare.

Ethical Statement: The authors are accountable for all aspects of the work in ensuring that questions related to the accuracy or integrity of any part of the work are 
appropriately investigated and resolved.

Open Access Statement: This is an Open Access article distributed in accordance with the Creative Commons Attribution-NonCommercial-NoDerivs 4.0 International License (CC BY-NC-ND 4.0), which permits the noncommercial replication and distribution of the article with the strict proviso that no changes or edits are made and the original work is properly cited (including links to both the formal publication through the relevant DOI and the license). See: https://creativecommons.org/licenses/by-nc-nd/4.0/.

\section{References}

1. Thomson JA, Itskovitz-Eldor J, Shapiro SS, et al. Embryonic stem cell lines derived from human blastocysts. Science 1998;282:1145-7.

2. Chung YG, Eum JH, Lee JE, et al. Human somatic cell nuclear transfer using adult cells. Cell Stem Cell 2014;14:777-80.

3. Sharma R, Khristov V, Rising A, et al. Clinical-grade stem cell-derived retinal pigment epithelium patch rescues retinal degeneration in rodents and pigs. Sci Transl Med 2019;11:eaat5580.

4. Li Y, Hermanson DL, Moriarity BS, et al. Human iPSCDerived Natural Killer Cells Engineered with Chimeric Antigen Receptors Enhance Anti-tumor Activity. Cell Stem Cell 2018;23:181-192.e5.

5. Sugimura R, Jha DK, Han A, et al. Haematopoietic stem and progenitor cells from human pluripotent stem cells. Nature 2017;545:432-8.

6. Kennedy M, Awong G, Sturgeon CM, et al. T lymphocyte potential marks the emergence of definitive hematopoietic progenitors in human pluripotent stem cell differentiation cultures. Cell Rep 2012;2:1722-35.

7. Baxter M, Withey S, Harrison S, et al. Phenotypic and functional analyses show stem cell-derived hepatocytelike cells better mimic fetal rather than adult hepatocytes. J Hepatol 2015;62:581-9.

8. Veres A, Faust AL, Bushnell HL, et al. Charting cellular identity during human in vitro $\beta$-cell differentiation. Nature 2019;569:368-73.

9. Pagliuca FW, Millman JR, Gürtler M, et al. Generation of functional human pancreatic $\beta$ cells in vitro. Cell 2014;159:428-39.

10. D'Amour KA, Bang AG, Eliazer S, et al. Production of pancreatic hormone-expressing endocrine cells from human embryonic stem cells. Nat Biotechnol
2006;24:1392-401.

11. Kobayashi T, Yamaguchi T, Hamanaka S, et al. Generation of rat pancreas in mouse by interspecific blastocyst injection of pluripotent stem cells. Cell 2010;142:787-99.

12. Chen J, Lansford R, Stewart V, et al. RAG-2-deficient blastocyst complementation: an assay of gene function in lymphocyte development. Proc Natl Acad Sci U S A 1993;90:4528-32.

13. Tsai FY, Keller G, Kuo FC, et al. An early haematopoietic defect in mice lacking the transcription factor GATA-2. Nature 1994;371:221-6.

14. Stanger BZ, Tanaka AJ, Melton DA. Organ size is limited by the number of embryonic progenitor cells in the pancreas but not the liver. Nature 2007;445:886-91.

15. Espejel S, Roll GR, McLaughlin KJ, et al. Induced pluripotent stem cell-derived hepatocytes have the functional and proliferative capabilities needed for liver regeneration in mice. J Clin Invest 2010;120:3120-6.

16. Yamaguchi T, Sato H, Kato-Itoh M, et al. Interspecies organogenesis generates autologous functional islets. Nature 2017;542:191-6.

17. Isotani A, Hatayama H, Kaseda K, et al. Formation of a thymus from rat ES cells in xenogeneic nude mouse $\leftrightarrow$ rat ES chimeras. Genes Cells 2011;16:397-405.

18. Tesar PJ, Chenoweth JG, Brook FA, et al. New cell lines from mouse epiblast share defining features with human embryonic stem cells. Nature 2007;448:196-9.

19. Huang Y, Osorno R, Tsakiridis A, et al. In Vivo differentiation potential of epiblast stem cells revealed by chimeric embryo formation. Cell Rep 2012;2:1571-8.

20. Mascetti VL, Pedersen RA. Human-Mouse Chimerism Validates Human Stem Cell Pluripotency. Cell Stem Cell 2016;18:67-72.

21. Gafni O, Weinberger L, Mansour AA, et al. Derivation of novel human ground state naive pluripotent stem cells. Nature 2013;504:282-6.

22. Takashima Y, Guo G, Loos R, et al. Resetting transcription factor control circuitry toward ground-state pluripotency in human. Cell 2014;158:1254-69.

23. Theunissen TW, Friedli M, He Y, et al. Molecular Criteria for Defining the Naive Human Pluripotent State. Cell Stem Cell 2016;19:502-15.

24. Theunissen TW, Powell BE, Wang H, et al. Systematic identification of culture conditions for induction and maintenance of naive human pluripotency. Cell Stem Cell 2014;15:471-87.

25. Wu J, Platero-Luengo A, Sakurai M, et al. Interspecies Chimerism with Mammalian Pluripotent Stem Cells. Cell 
2017;168:473-486.e15.

26. Yang Y, Liu B, Xu J, et al. Derivation of Pluripotent Stem Cells with In Vivo Embryonic and Extraembryonic Potency. Cell 2017;169:243-257.e25.

27. Hu Z, Li H, Jiang H, et al. Transient inhibition of mTOR in human pluripotent stem cells enables robust formation of mouse-human chimeric embryos. Sci Adv 2020;6:eaaz0298.

28. Masaki H, Kato-Itoh M, Takahashi Y, et al. Inhibition of Apoptosis Overcomes Stage-Related Compatibility Barriers to Chimera Formation in Mouse Embryos. Cell Stem Cell 2016;19:587-92.

29. Wang X, Li T, Cui T, et al. Human embryonic stem cells contribute to embryonic and extraembryonic lineages in mouse embryos upon inhibition of apoptosis. Cell Res 2018;28:126-9.

30. Huang K, Zhu Y, Ma Y, et al. BMI1 enables interspecies chimerism with human pluripotent stem cells. Nat Commun 2018;9:4649.

31. Zheng C, Hu Y, Sakurai M, et al. Cell competition constitutes a barrier for interspecies chimerism. Nature 2021;592:272-6.

32. Das S, Koyano-Nakagawa N, Gafni O, et al. Generation of human endothelium in pig embryos deficient in ETV2. Nat Biotechnol 2020;38:297-302.

33. Ballard EB, Wu J. Growth Competition in Interspecies Chimeras: A New Paradigm for Blastocyst Complementation. Cell Stem Cell 2021;28:3-5.

34. Nishimura T, Suchy FP, Bhadury J, et al. Generation of Functional Organs Using a Cell-Competitive Niche in Intra- and Inter-species Rodent Chimeras. Cell Stem Cell 2021;28:141-149.e3.

35. Hamanaka S, Umino A, Sato H, et al. Generation of Vascular Endothelial Cells and Hematopoietic Cells by Blastocyst Complementation. Stem Cell Reports 2018;11:988-97.

36. Oosting M, Cheng SC, Bolscher JM, et al. Human TLR10 is an anti-inflammatory pattern-recognition receptor. Proc

doi: $10.21037 /$ sci-2020-074

Cite this article as: Li Y, Huang K. Human-animal interspecies chimerism via blastocyst complementation: advances, challenges and perspectives: a narrative review. Stem Cell Investig 2021;8:20.
Natl Acad Sci U S A 2014;111:E4478-84.

37. Dahéron L, Opitz SL, Zaehres H, et al. LIF/STAT3 signaling fails to maintain self-renewal of human embryonic stem cells. Stem Cells 2004;22:770-8.

38. Zhang B, Ge B, Xia X, et al. Compatibility of porcine and human interleukin 2: implications for xenotransplantation. Xenotransplantation 2006;13:423-32.

39. Brehm MA, Racki WJ, Leif J, et al. Engraftment of human HSCs in nonirradiated newborn NOD-scid IL2r $\gamma$ null mice is enhanced by transgenic expression of membranebound human SCF. Blood 2012;119:2778-88.

40. Takagi S, Saito Y, Hijikata A, et al. Membrane-bound human SCF/KL promotes in vivo human hematopoietic engraftment and myeloid differentiation. Blood 2012;119:2768-77.

41. Rongvaux A, Willinger T, Takizawa H, et al. Human thrombopoietin knockin mice efficiently support human hematopoiesis in vivo. Proc Natl Acad Sci U S A 2011;108:2378-83.

42. Rongvaux A, Willinger T, Martinek J, et al. Development and function of human innate immune cells in a humanized mouse model. Nat Biotechnol 2014;32:364-72.

43. Song Y, Shan L, Gbyli R, et al. Combined liver-cytokine humanization comes to the rescue of circulating human red blood cells. Science 2021;371:1019-25.

44. Wu J, Platero Luengo A, Gil MA, et al. Generation of human organs in pigs via interspecies blastocyst complementation. Reprod Domest Anim 2016;51 Suppl 2:18-24.

45. Yamaguchi T. Hurdles to Generating Human Islets in Animals via Blastocyst Complementation. Curr Diab Rep 2019; $19: 45$.

46. Lu Y, Zhou Y, Ju R, et al. Human-animal chimeras for autologous organ transplantation: technological advances and future perspectives. Ann Transl Med 2019;7:576.

47. Niu D, Wei HJ, Lin L, et al. Inactivation of porcine endogenous retrovirus in pigs using CRISPR-Cas9. Transgenic Research 2018;27:483. 